\title{
Cut and Paste: restoring cellular function by gene correction
}

\author{
Guang-Hui Liu ${ }^{1,2}$, Ignacio Sancho-Martinez ${ }^{1}$, Juan Carlos Izpisua Belmonte ${ }^{1,3}$ \\ ${ }^{1}$ Gene Expression Laboratory, Salk Institute for Biological Studies, 10010 North Torrey Pines Road, La Jolla, California 92037, \\ USA; ${ }^{2}$ National Laboratory of Biomacromolecules, Institute of Biophysics, Chinese Academy of Sciences, Beijing 100101, China; \\ ${ }^{3}$ Center for Regenerative Medicine in Barcelona, Dr. Aiguader 88, 08003 Barcelona, Spain \\ Cell Research (2012) 22:283-284. doi:10.1038/cr.2011.192; published online 6 December 2011
}

Gene-editing technologies and patient-specific induced pluripotent stem cells (iPSCs) may represent an unprecedented opportunity for merging the stem cell and traditional gene therapy fields to fulfill the promises of regenerative medicine.

Gene correction technologies may allow for the precise excision of mutant genes responsible for monogenic disease and further replacement with its wild-type version. When combined with the use of pluripotent cells, gene correction approaches could ultimately lead to the cure or alleviation of human disease. Jaenisch and colleagues published the first demonstration of phenotypic improvement upon gene correction in 2007 [1]. By using a mouse model of sickle cell anemia, the authors were able to "edit" the genome of murine iPSCs, thereby allowing for the re-introduction of a wild-type version of the defective hemoglobin gene. Upon transplantation into the mouse model, hematopoietic progenitor cells derived from corrected iPSCs led to normal erythrocyte function. Subsequently, two different reports demonstrated the phenotypic correction of Hemophilia A by either allogeneic transplantation of endothelial cells derived from wildtype iPSCs or by in vivo correction of

Correspondence: Juan Carlos Izpisua Belmonte E-mail: belmonte@salk.edu; izpisua@cmrb.eu the mutant factor VIII $[2,3]$. The studies by Xu et al. did not involve genetic correction of the generated iPSCs, but were rather a proof-of-principle application of wild-type iPSC differentiated cells as a "dominant positive" graft alleviating the disease phenotype [2]. On the other hand, the approach by Li et al. involved Zinc Finger Nuclease (ZFN)-mediated in vivo correction and alleviation of the hemophilic phenotype without iPSC generation or cell transplantation [3]. The ZFN-mediated correction of Hemophilia, although a more "canonical" gene therapy approach, actually showed efficient targeting, splicing and replacement with the wild-type version of the mutated DNA fragment in vivo.

More recently, different research groups have demonstrated the successful generation and correction of patient-specific iPSCs. They include the progeria syndrome [4, 5], Parkinson's disease [6], gyrate atrophy [7] as well as hematological diseases such as Thalassemias [8], Fanconi Anemia [9], and sickle cell anemia [14]. Even though these studies lacked in vivo confirmation of the phenotypic correction, some of them demonstrated functional correction of the phenotype upon differentiation in vitro. Thus, in vitro differentiation of pluripotent cells seems to mimic physiological processes, accelerate the time required for symptomatic manifestation of the disease in vitro and accordingly represent a suitable platform for gene correction studies. Of note, methods based on the application of Helper-dependent Adenoviral Vectors (HDAdVs) have proven successful with editing large genomic regions, potentially allowing for the single-step correction by homologous recombination of genes bearing multiple point mutations or even certain deletions and duplications [5, 14]. Most importantly, even though different methods were used in the various reports, gene targeting itself did not seem to affect epi/ genomic integrity $[5-7,14]$. Thus, the broad range of applications for gene editing technologies may not only be circumscribed to gene correction and regenerative medicine, but it could also allow for concise analysis of the molecular mechanisms leading to disease manifestation and progression.

Taking into account previously described epigenetic as well as genetic abnormalities found in iPSCs, the use of ESCs could prove a suitable complement not only for safer gene correction strategies, but also for cell's modification in the opposite direction, the generation of disease-specific ESCs $[10,11]$. Such an approach, based on the use of site-specific nucleases, has been recently demonstrated. Hockemeyer et al. have described for the first time the use of Transcription Activatorlike Effector Nucleases (TALE) for gene editing purposes in humans [12]. TALEN are site-specific nucleases that 
can be engineered similarly to ZFNs for the targeting of site-specific locations. The report describes the use of these novel nucleases for the efficient targeting of up to five different loci in both ESCs and iPSCs [12]. Importantly, the efficiencies described as well as the specificity shown for these nucleases were comparable to those achieved by employing more traditional methodologies such as ZFNs. Along the same line, Soldner et al. have reported the editing of the $\alpha$-synuclein gene, whose different variants have been linked to Parkinson susceptibility [6]. In this work the authors do not only described the efficient engineering and correction of this gene by ZFNs in Parkinson patientderived iPSCs, but also demonstrated the generation of Parkinson-specific ESCs lines [6].

More recently, Yusa et al. described the development of a novel gene targeting approach by combining the use of ZFN and piggyBAC transposon technology [13]. In their approach the authors were able to excise back the targeting construct from the host genome in the absence of the typical genomic scars induced by other commonly employed excisable methods such as the Cre-LoxP system. Of note are the facts that correction of iPSCs generated by non-integrative approaches was equally efficient as compared to the correction of iPSCs generated by integrative viruses and that the frequency for biallelic correction reaches up to $11 \%$ in some of the corrected clones. Moreover, even though the generated iPSCs and their derivatives bear certain mutations acquired during reprogramming, gene targeting and/or culture conditions, the mutations observed did not hamper the phenotypic correction in vivo, neither led to tumor formation. This work as well as several other reports published earlier this year highlight the need for exhaustive testing of iPSCs and their derivatives in the context of specific applications rather than absolute number of mutations acquired, i.e., iPSCs bearing mutations in genes compromising the function of differentiated cells should be discarded in favor of lines whose alterations do not hamper normal cell function upon differentiation and transplantation [13]. With these criteria in mind, one would rather prime functionality in a particular context instead of the absolute number of mutations observed.

The genetic correction of monogenic mutations responsible for the development of disease and generation of isogenic iPSC/ESC lines may not only contribute towards more reliable and experimentally matched control sets in future drug discovery studies, but also brings the opportunity for combining gene therapy and regenerative medicine in novel therapeutical treatments. Although these two fields of study are relatively recent, the astonishingly rapid pace of new development leads us to assume that our long-lasting bench dreams might become real, allowing us to witness one of the most revolutionary ages in personalized medicine.

\section{Acknowledgments}

Work in the laboratory of JCIB was supported by Sanofi-Aventis, The Helmsley Charitable Trust and The Ellison Medical Foundation.

\section{References}

1 Hanna J, Wernig M, Markoulaki S, et al. Treatment of sickle cell anemia mouse model with iPS cells generated from autologous skin. Science 2007; 318:1920-1923.

$2 \mathrm{Xu}$ D, Alipio Z, Fink LM, et al. Phenotypic correction of murine hemophilia A using an iPS cell-based therapy. Proc Natl Acad Sci USA 2009; 106:808813.

3 Li H, Haurigot V, Doyon Y, et al. In vivo genome editing restores haemosta- sis in a mouse model of haemophilia. Nature 2011; 475:217-221.

4 Liu GH et al. Recapitulation of premature ageing with iPSCs from Hutchinson-Gilford progeria syndrome. Nature 2011; 472:221-225.

5 Liu GH, Suzuki K, Qu J, et al. Targeted gene correction of laminopathyassociated LMNA mutations in patientspecific iPSCs. Cell Stem Cell 2011; 8:688-694.

6 Soldner F, Laganière J, Cheng AW, et al. Generation of isogenic pluripotent stem cells differing exclusively at two early onset Parkinson point mutations. Cell 2011; 146:318-331.

7 Howden SE, Gore A, Li Z, et al. Genetic correction and analysis of induced pluripotent stem cells from a patient with gyrate atrophy. Proc Natl Acad Sci USA 2011; 108:6537-6542.

8 Papapetrou EP, Lee G, Malani N, et al. Genomic safe harbors permit high $\beta$-globin transgene expression in thalassemia induced pluripotent stem cells. Nat Biotechnol 2011; 29:73-78.

9 Raya A, Rodríguez-Pizà I, Guenechea $\mathrm{G}$, et al. Disease-corrected haematopoietic progenitors from Fanconi anaemia induced pluripotent stem cells. Nature 2009; 460:53-59.

10 Panopoulos AD, Ruiz S, Izpisua Belmonte JC. iPSCs: induced back to controversy. Cell Stem Cell 2011; 8:347348.

11 Urbach A, Bar-Nur O, Daley GQ, Benvenisty N. Differential modeling of fragile $\mathrm{X}$ syndrome by human embryonic stem cells and induced pluripotent stem cells. Cell Stem Cell 2010; 6:407411.

12 Hockemeyer D, Wang H, Kiani S, et al. Genetic engineering of human pluripotent cells using TALE nucleases. Nat Biotechnol 2011; 29:731-734.

13 Yusa K, Rashid ST, Strick-Marchand $\mathrm{H}$, et al. Targeted gene correction of $\alpha 1$-antitrypsin deficiency in induced pluripotent stem cells. Nature 2011; 478:391-394.

14 Li M, Suzuki K, Qu J, et al. Efficient correction of hemoglobinopathy-causing mutations by homologous recombination in integration-free patient iPSCs. Cell Res 2011; 21:1740-1744. 